\title{
Instantaneous fluid force acting on a circular cylinder control by a small rod
}

\author{
Takayuki Tsutsui ${ }^{1, *}$ \\ ${ }^{1}$ Department of Mechanical Engineering, The National Defense Academy, 1-10-20 Hashirimizu, Yokosuka, Kanagawa 238-8686 Japan
}

\begin{abstract}
Two unique bluff body flow control methods using a small rod have been proposed in previous studies. The first is the forced reattachment method, which is a type of separated shear layer control. This method reduces drag and generates a lift. The second is the front rod method, which involves the placement of a small rod upstream of the bluff body to control the incoming flow and reduce drag. This paper describes the features of the instantaneous fluid force achieved using these flow control methods. These methods were experimentally applied to a cylinder, and the instantaneous pressure field and the flow visualizations of these methods are presented. When the forced reattachment method was applied, a lift force was generated, and the vibration was less than that in the case of the front rod method. When the front rod method was applied, the drag force was reduced by over $50 \%$ in comparison with those in the uncontrolled case and the case with the forced reattachment method.
\end{abstract}

\section{Introduction}

The ability to manipulate a flow field is of immense technological importance. For example, if drag of vehicles and buildings can be reduced, much fuel cost and construction materials for buildings would be saved. Flow control around bluff bodies is of importance and of interest for engineering field.

To investigate flow control around a circular cylinder, two previously proposed flow control methods using a small rod were applied [ $1-9]$. The first is the forced reattachment method [1 - 4]. Figure 1 shows a visualization of the flow and the distribution of the pressure coefficient $\mathrm{C}_{\mathrm{p}}$ around a circular cylinder with forced reattachment flow control. This method achieves shear layer separation and reattachment using a small rod. Using this method reduced the drag of the circular cylinder by $20-30 \%$ and generated a lift coefficient $C_{L}$ of 1.0 [1]. In this method, the separated shear layer from the upper side of the cylinder adheres to the rear of the cylinder as a result of the control by the small rod, causing a stagnant region to form behind the cylinder. The $C_{p}$ distribution on the lower side is higher than that without a small rod, thus generating a lift.

The second method is the front rod method. In this method, a small rod is placed upstream of a bluff body. When this method is applied to a circular cylinder, it has been reported that the total drag coefficient is reduced by $63 \%$ relative to that in the uncontrolled case $[5,6]$. Figure 2 shows the flow visualization and the $C_{p}$ distribution around the circular cylinder for this method. This method has been applied to bluff bodies of several shapes: a flat plate [7], a square prism [8], and an inclined square prism [9]. In these cases, the total drag coefficients were decreased by $34-75 \%$ relative to that without the rod.

The general features, such as the static and timeaveraged field fluid forces, of the two flow control methods have been previously reported. However, these studies did not report the instantaneous fluid force acting on cylinders. The oscillation of the fluid force can sometimes cause vibrations in civil engineering structures. It is therefore important to understand the instantaneous features of these flow control methods.

The aim of this study was to describe the instantaneous behaviors of a flow around a circular cylinder under these flow control methods. This paper presents the instantaneous pressure field and flow visualizations obtained using these two methods. From these results, the features of the oscillating drag and lift were clarified.

\section{Experimental apparatus and methods}

The coordinate systems and notation used in the present study for the forced reattachment and front rod methods are shown in Figure 3(a) and (b), respectively. For the forced reattachment method, the diameters of the circular cylinder and small rod were $\mathrm{D}=40 \mathrm{~mm}$ and $\mathrm{d}=10 \mathrm{~mm}$, respectively. The small rod was placed in the shear layer of the cylinder. The gap between the cylinder and small rod had a width of $\mathrm{G}=4 \mathrm{~mm}$, and the angular position of the rod was $\alpha=125^{\circ}$. The forced reattachment phenomenon can be induced by a rod as small as $2 \mathrm{~mm}$ in diameter [ $1-4]$; however, in this study, to ensure the steady occurrence of the forced reattachment phenomenon, the diameter of the small rod was $10 \mathrm{~mm}$.

For the front rod method, the diameters of the 
circular cylinder and small rod were $\mathrm{D}=40 \mathrm{~mm}$ and $\mathrm{d}=$ $10 \mathrm{~mm}$, respectively. The small rod was placed upstream of the cylinder. The longitudinal distance between the axes of the cylinder and small rod was $\mathrm{L}=70 \mathrm{~mm}$.

Figure 4 shows a schematic of the wind tunnel and pressure measurement equipment. The experiments were performed in a low-speed wind tunnel, the working section of which has a height of $1,000 \mathrm{~mm}$, a width of $150 \mathrm{~mm}$, and a length of $1,200 \mathrm{~mm}$. The free-stream velocity $U$ was varied from 6 to $20 \mathrm{~m} / \mathrm{s}$, and the turbulent intensity was approximately $0.4 \%$ in this velocity range. The Reynolds number based on $\mathrm{D}$ ranged from $3.8 \times 10^{4}$ to $1.3 \times 10^{5}$. The velocity fluctuation in the wake and the time-averaged and instantaneous pressures on the surfaces of the cylinders were measured using this setup. Flow visualization was performed using the smoke-wire method.

A detailed view of the circular cylinder is shown in Figure 5(a). The cylinder is made of acrylic resin and has two pressure taps of $1.6 \mathrm{~mm}$ in diameter connected to a manometer and a semi-conductor pressure sensor. The pressure distribution over the entire surface of the cylinder can be determined by rotating it about its center axis.
Figure 5(b) shows the measurement technique used to measure the instantaneous pressure around the circular cylinder. The velocity fluctuating signal was used as a standard signal to align the phase. A hot-wire probe was placed behind the cylinder to measure the vortex shedding frequency. The hot wire was placed at a radial distance of $\mathrm{D}$ and a downstream distance of 3D from the center of the cylinder. The pressure signals at $\phi$ were recorded simultaneously while rotating the cylinders by increments of $10^{\circ}$. The oscillating pressure distribution was obtained from the instantaneous pressure coefficients measured at the same phase for a given cylinder rotation angle $\phi$.

Flow visualization was conducted using the smokewire method. Figure 6 shows a schematic diagram of the smoke-wire system. The bottom of the fluctuating velocity signal is the trigger position for flashing the stroboscope. Images of the flow around the cylinders can be captured at any phase by setting the delay time.

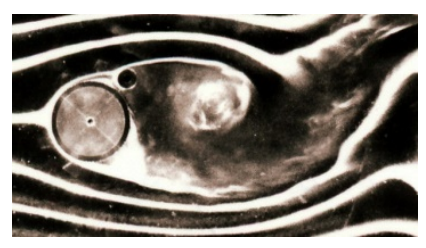

(a) flow visualization $\left(\mathrm{Re}=1 \times 10^{4}\right)$ (b) time averaged pressure distribution $\left(\mathrm{Re}=4.2 \times 10^{4}\right)$

Fig. 1 . Flow controlled by a small rod (forced reattachment method, $D=40 \mathrm{~mm}, \mathrm{~d}=10 \mathrm{~mm}, \mathrm{G}=4 \mathrm{~mm}, \alpha=125^{\circ}$ )

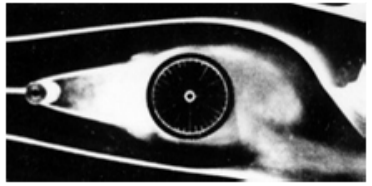

(a) flow visualization $\left(\operatorname{Re}=1 \times 10^{4}\right)$

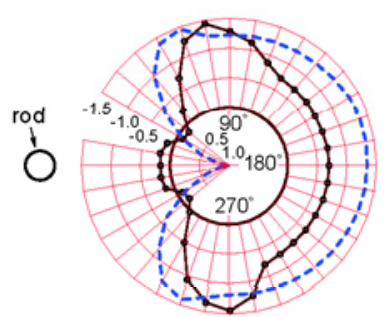

(b) time averaged pressure distribution $\left(\operatorname{Re}=4.2 \times 10^{4}\right)$

Fig.2. Flow controlled by a small rod (front rod method, $D=40 \mathrm{~mm}, \mathrm{~d}=10 \mathrm{~mm}, \mathrm{~L}=70 \mathrm{~mm}$ )

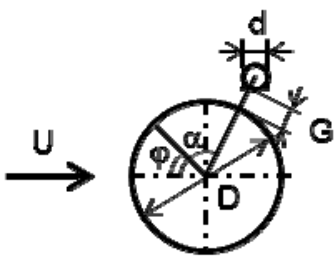

(a) forced reattachment method

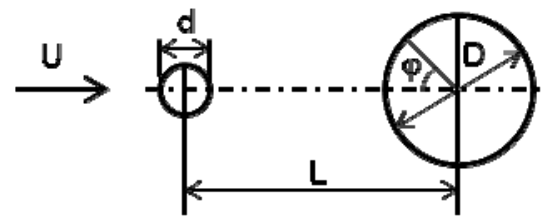

(b) front rod method

Fig. 3. Corrdinate system and notation 


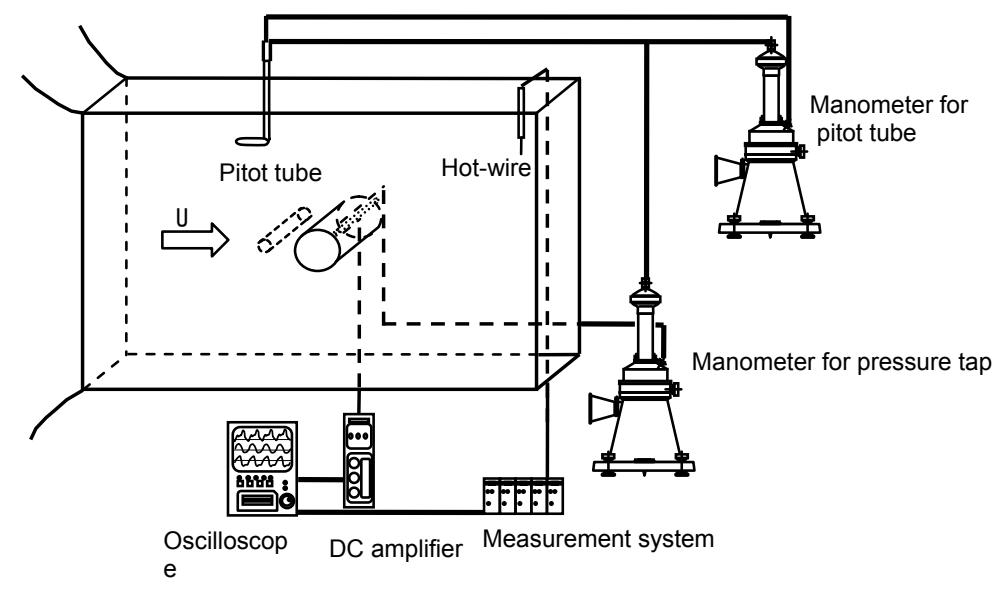

Fig. 4. Pressure measurement system

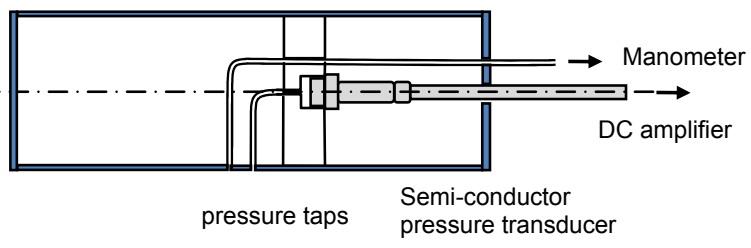

(a) pressure measurement model

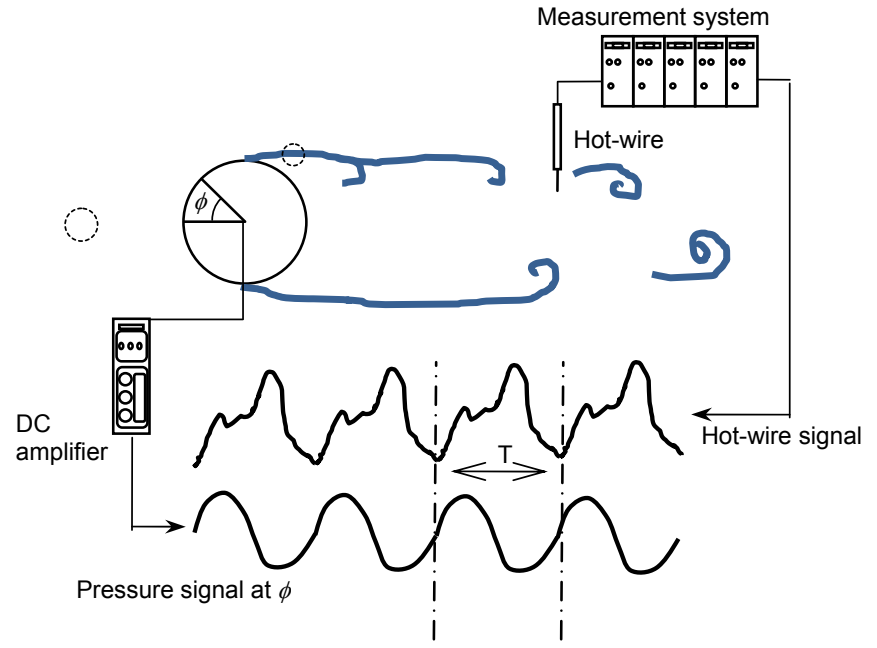

(b) instantaneous pressure measurement technique

Fig. 5. Pressure measurement technique

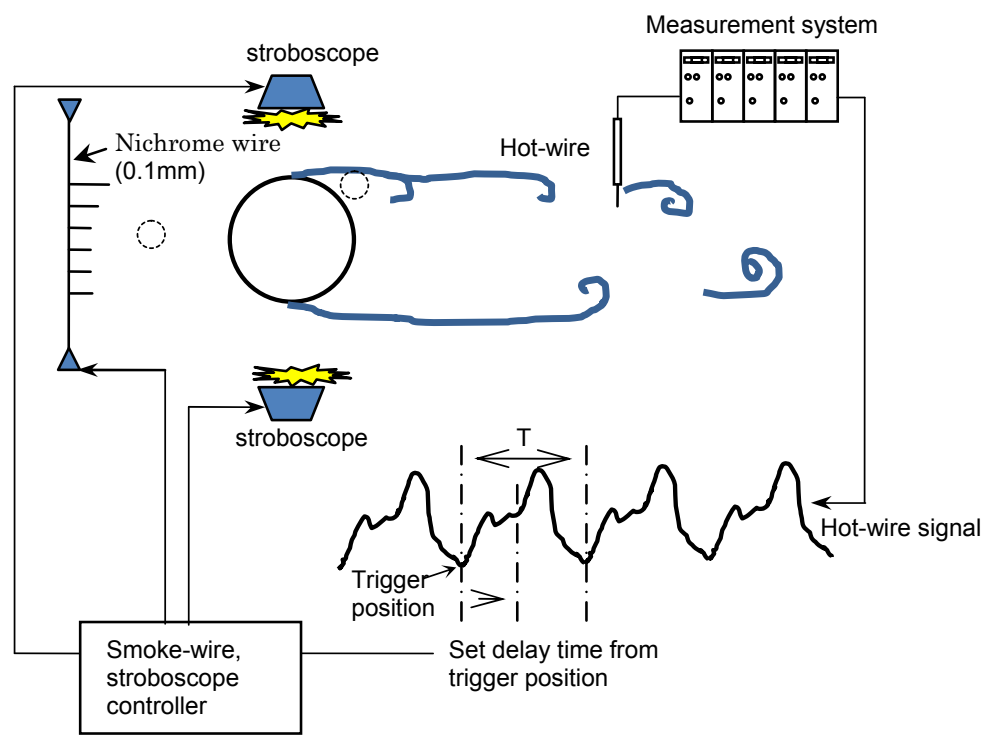

Fig. 6. Flow visualization technique 


\section{Results and discussion}

\subsection{Single cylinder}

To confirm the validity of the measurement methods, the flow was visualized, and the time-averaged and instantaneous pressures over a circular cylinder by a small rod were measured. Figure 7 shows flow visualization photographs of the circular cylinder without a small rod from $\mathrm{t} / \mathrm{T}=0$ to $7 / 8$ at intervals of 1 / 8 , where $\mathrm{T}$ and $\mathrm{t}$ are the period of vortex shedding and the time, respectively. The Strouhal number $\mathrm{St}$ is defined as $f D / U$, where $f$ is the vortex shedding frequency; in this experiment, a Strouhal number was $\mathrm{St}=0.20$.
The instantaneous pressure distributions at different stages of the vortex shedding cycle are shown in Fig. 8. Negative and positive pressures are plotted inside and outside of the circle, respectively. The motion of the flow around a bluff body, such as a cylinder, is a periodic motion that regularly returns to a given position. These pressure distributions correspond to the flow visualization photographs in Figure 7.

The oscillating drag and lift coefficients $\mathrm{CD}_{\mathrm{D}}$ and $\mathrm{CL}_{\mathrm{L}}$ of the circular cylinder obtained in the present study and are shown in Figure 9 along with those obtained in a previous study by Nishimura and Taniike [10]. The drag and lift coefficients were obtained by integrating the pressure distribution. The present results show the same tendencies as the previous results.

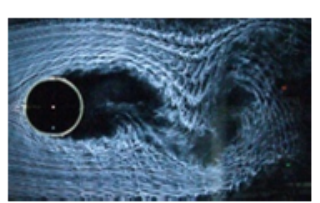

(a) $t / T=0 / 8$

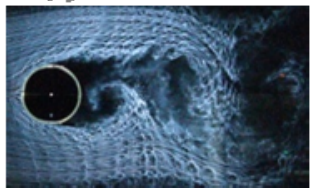

(b) $1 / 8$

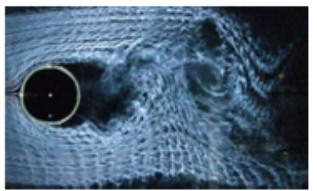

(c) $2 / 8$

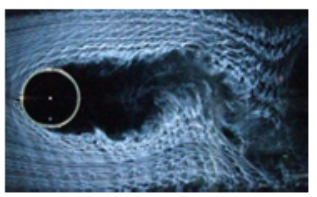

(g) $6 / 8$

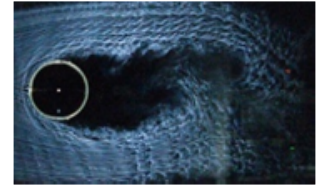

(d) $3 / 8$

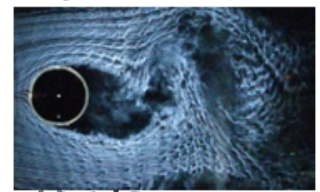

(e) $4 / 8$

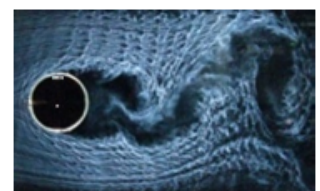

(f) $5 / 8$

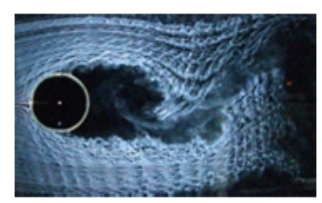

(h) $7 / 8$

Fig. 7. Sequential photographs of a single circular cylinder $\left(\mathrm{D}=40 \mathrm{~mm}, \mathrm{Re}=4.2 \times 10^{4}, \mathrm{St}=0.20, \mathrm{f}\right.$ $=80 \mathrm{~Hz}, \mathrm{~T}=12.5 \mathrm{~ms}$ )

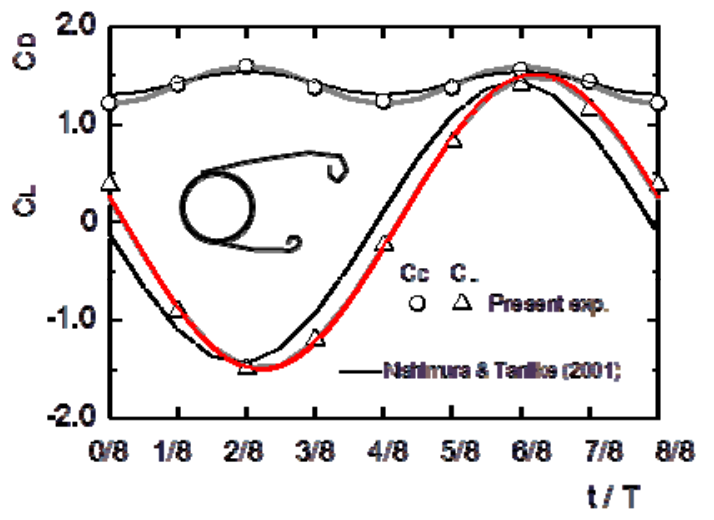

Fig. 9. Oscillating drag and lift coefficient of a single circular cylinder

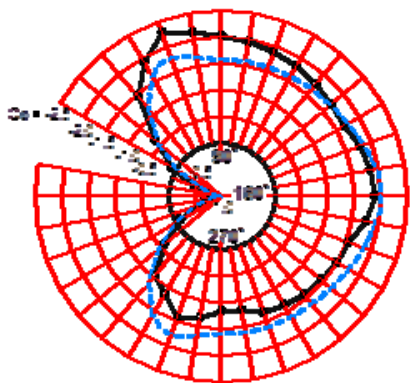

(a) $\mathrm{t} / \mathrm{T}=0 / 8$---mases $\mathrm{c}_{\mathrm{f}}$

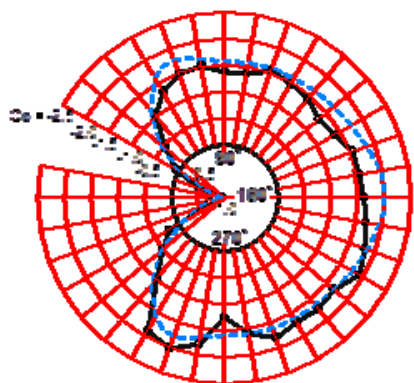

(d) $3 / 8$
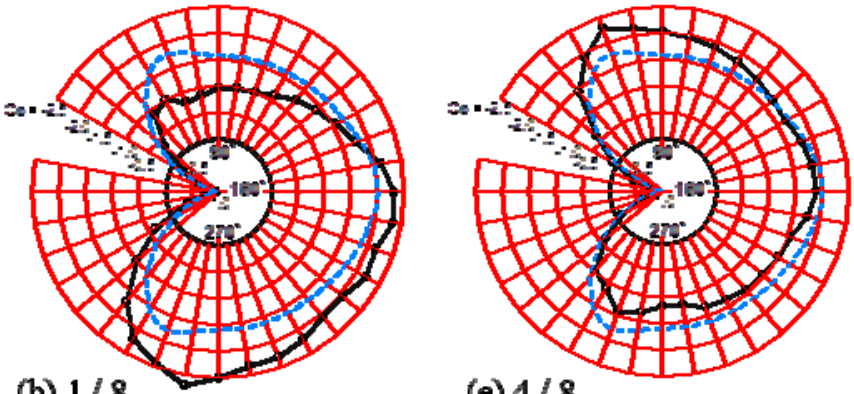

(e) $4 / 8$
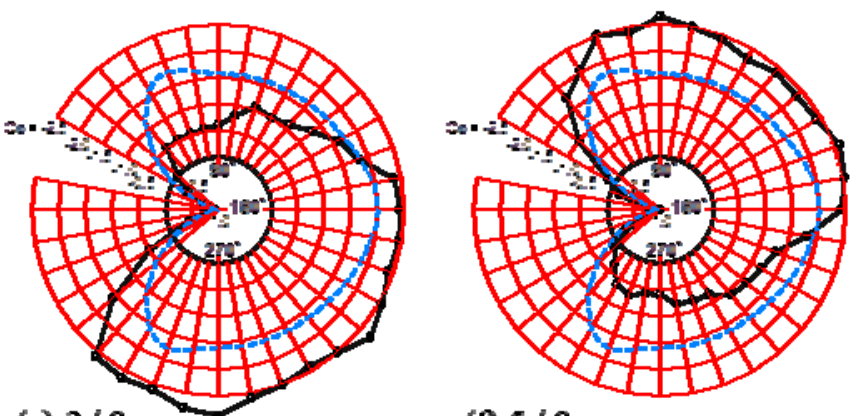

(c) $2 / 8$

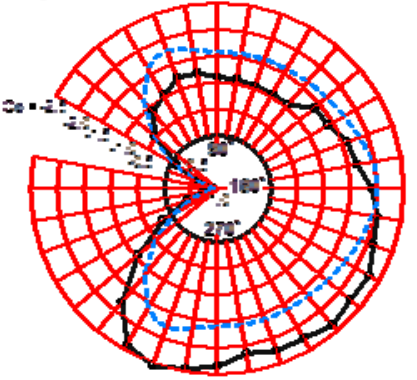

(g) $6 / 8$

(f) $5 / 8$

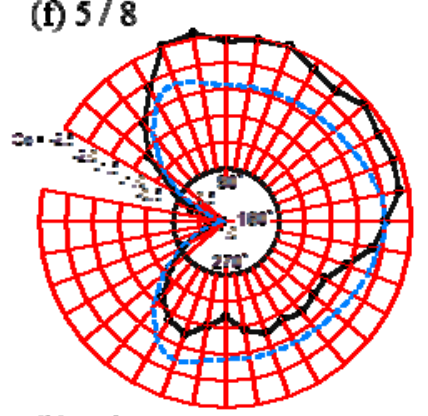

(h) $7 / 8$

Fig. 8. Instantaneous pressure distribution of a single circular cylinder at different stages of a vortex shedding cycle 


\subsection{Forced reattachment method}

First, the forced reattachment phenomenon was considered. Figure 10 shows a schematic of the forced reattachment phenomenon. The small rod was positioned just inside of the shear layer separated from the upper side of the circular cylinder so that it divided the shear layer. In this setup, the lower shear layer reattaches and adheres to the rear side of the cylinder, and the upper shear layer is supported and elongated by the small rod. A large stagnant region forms behind the cylinder. This forced reattachment phenomenon is not an intermittent phenomenon, and thus the wake motion is not more active than that of single circular cylinder. Under this control method, the mean pressure is asymmetrically distributed between the upper and lower sides of the cylinder. As a result, a lift force is generated.

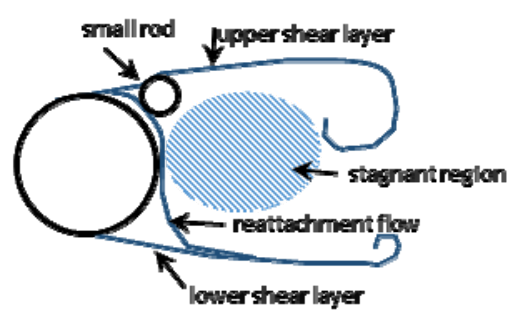

Fig. 10. Description of the forced reattachment phenomenon

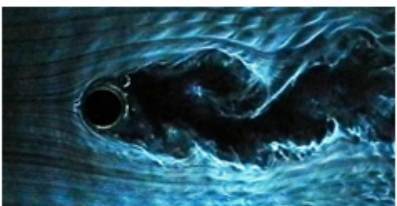

(a) $t / T=0 / 8$

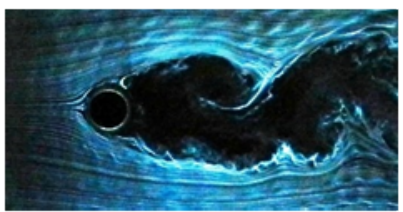

(b) $1 / 8$

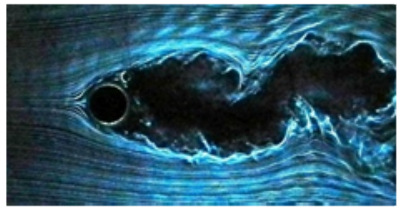

(c) $2 / 8$

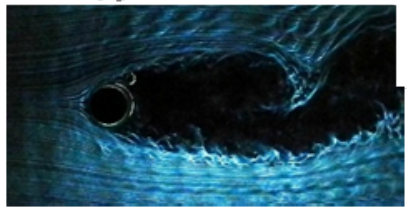

(g) $6 / 8$

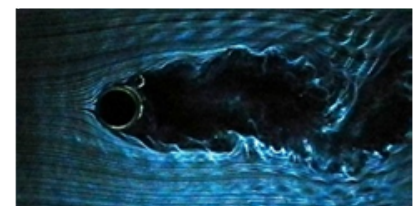

(d) $3 / 8$

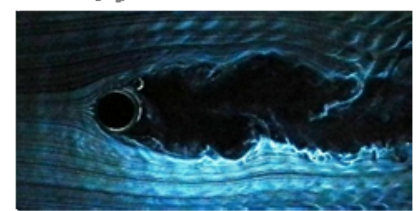

(e) $4 / 8$

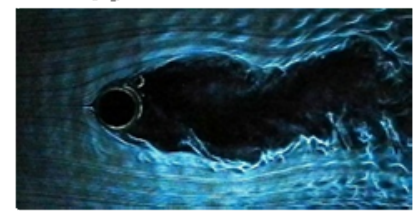

(f) $5 / 8$

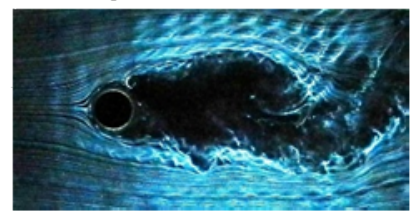

(h) $7 / 8$
Fig. 11. Sequential photographs of the controlled circular cylinder by the forced reattachment method $\left(\mathrm{D}=40 \mathrm{~mm}, \mathrm{~d}=10 \mathrm{~mm}, \mathrm{G}=4 \mathrm{~mm}, \alpha=125^{\circ}, \operatorname{Re}=4.2\right.$ $\times 10^{4}, \mathrm{St}=0.19, \mathrm{f}=74 \mathrm{~Hz}, \mathrm{~T}=13.5 \mathrm{~ms}$ )
Figure 11 shows flow visualization photographs at eight different stages of the vortex shedding cycle $(\mathrm{t} / \mathrm{T}$ $=0$ to $7 / 8$ at intervals of $1 / 8$ ) of the circular cylinder flow controlled by the forced reattachment method. The flow visualization photographs show that the upper and lower shear layers were elongated and the vortex street was not observed. This means that the wake motion is not more active than that of single circular cylinder, because the wake motion was constrained by the upper shear layer and the reattachment flow.

Figure 12 shows the instantaneous pressure distribution at eight different stages of the vortex shedding cycle $(\mathrm{t} / \mathrm{T}=0$ to $7 / 8$ at intervals of $1 / 8$ ) along with the time-averaged pressure distributions. At every value of $t / T$, the $C_{p}$ distribution was asymmetric about the horizontal center line, therefore, a lift acting in the upward direction was generated.

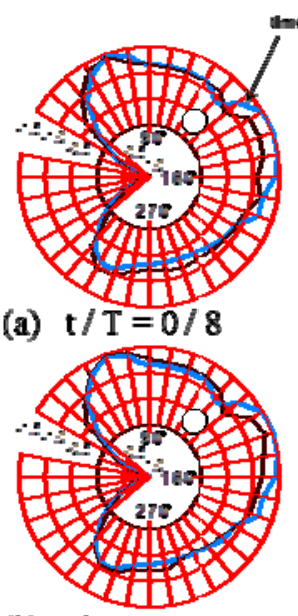

(b) $1 / 8$

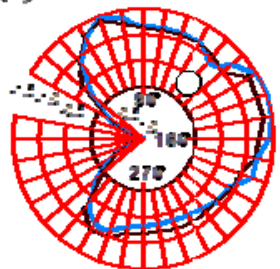

(c) $2 / 8$

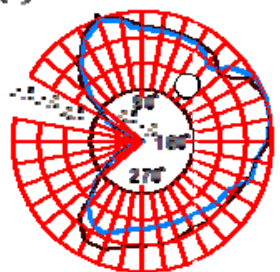

(g) $6 / 8$

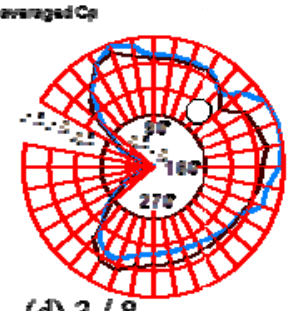

(d) $3 / 8$

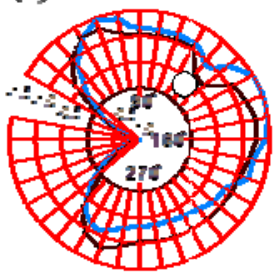

(e) $4 / 8$

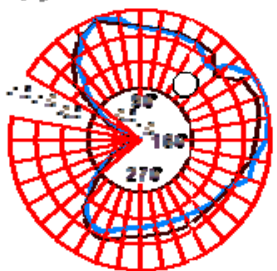

(f) $5 / 8$

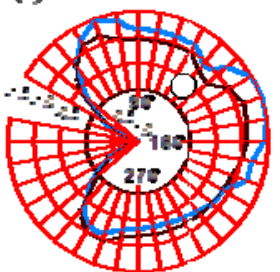

(h) $7 / 8$

Fig. 12. Instantaneous pressure distribution of the controlled circular cylinder at different stages of a vortex shedding cycle 


\subsection{Front rod method}

Figure 13 shows a brief schematic of the front rod method. In this setup, the shear layer separated from the small rod reattaches to the front side of the cylinder and a quasi-static vortex is formed between the rod and cylinder. A separation bubble forms, causing the separation point to move backward on the cylinder and the wake to decrease relative to that in the case with a single cylinder. Figure 14 shows flow visualization photographs of the cylinder flow controlled using the front rod method. A vortex street was clearly observed behind the cylinder.

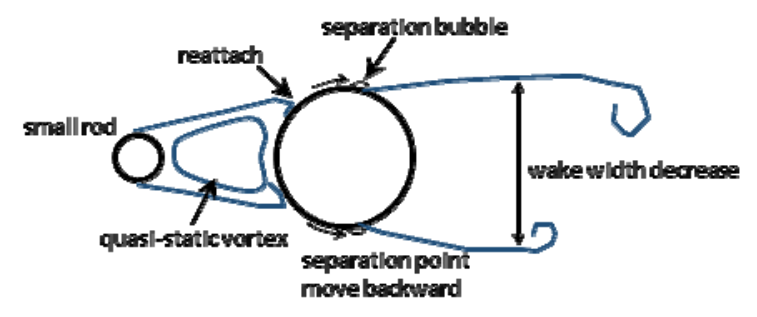

Fig. 13. Description of the flow control by a rod

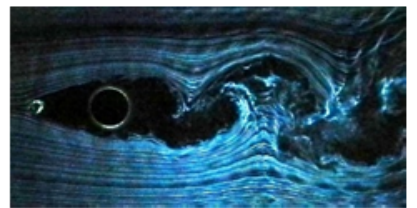

(a) $t / T=0 / 8$

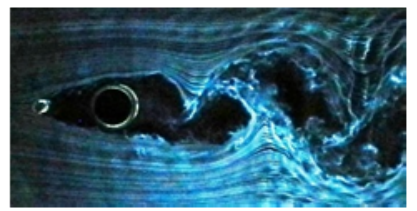

(b) $1 / 8$

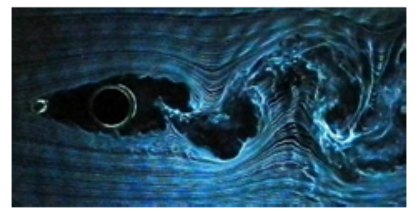

(c) $2 / 8$

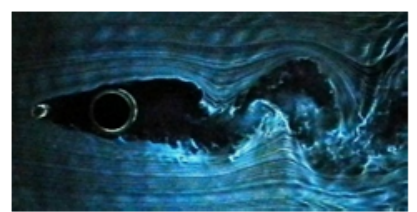

(g) $6 / 8$

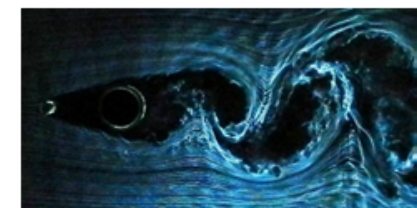

(d) $3 / 8$

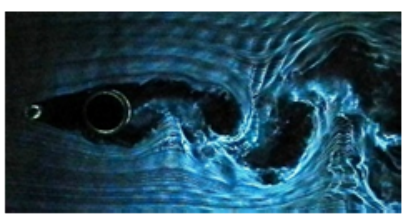

(e) $4 / 8$

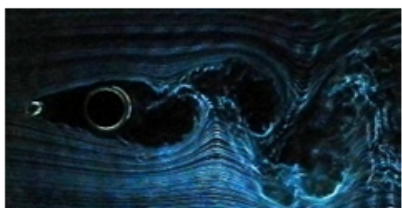

(f) $5 / 8$

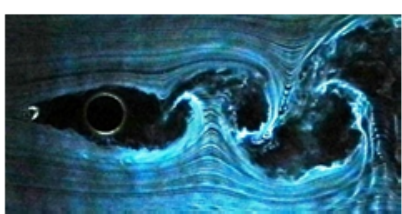

(h) $7 / 8$
Fig. 14. Sequential photographs of the controlled circular cylinder by the front rod method (D $=40 \mathrm{~mm}, \mathrm{~d}=10 \mathrm{~mm}, \mathrm{~L}=70 \mathrm{~mm}, \mathrm{Re}=4.2 \times 10^{4}, \mathrm{st}$ $=0.22, \mathrm{f}=90 \mathrm{~Hz}, \mathrm{~T}=11.1 \mathrm{~ms}$ )
The instantaneous pressure distributions for this case at eight different stages of the vortex shedding cycle $(\mathrm{t} /$ $\mathrm{T}=0$ to $7 / 8$ at intervals of $1 / 8$ ) are shown in Figure 15. The pressure is always negative in the front region, and $\mathrm{C}_{\mathrm{p}}$ has two maximum values at $\phi= \pm 30^{\circ}$, which are the reattachment points of the shear layer separated from the small rod. The $C_{p}$ value at the front side of the cylinder has negative value because of the quasi-static vortex. At every value of $\mathrm{t} / \mathrm{T}$, the back pressure is higher than the corresponding pressures obtained using the forced reattachment method and those of the single cylinder. This indicates that a reduction in the drag reduction can be expected.

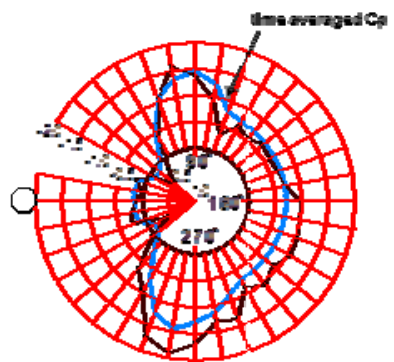

(a) $t / T=0 / 8$

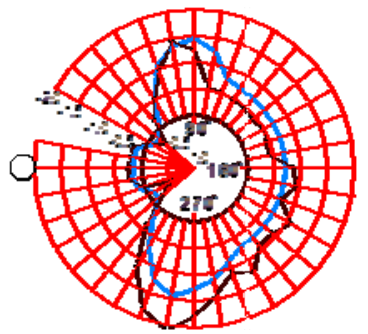

(b) $1 / 8$

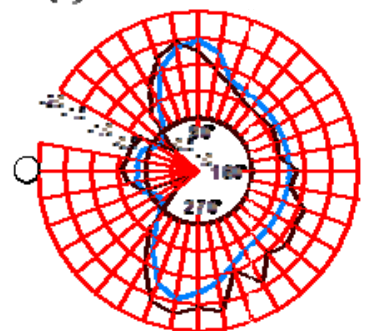

(c) $2 / 8$

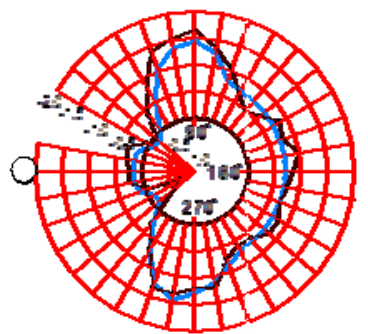

(g) $6 / 8$

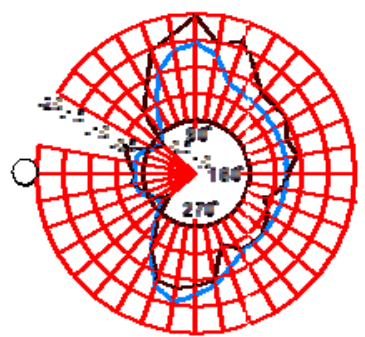

(d) $3 / 8$

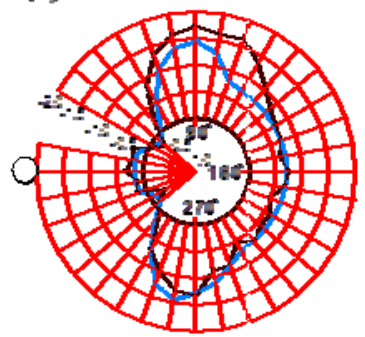

(e) $4 / 8$

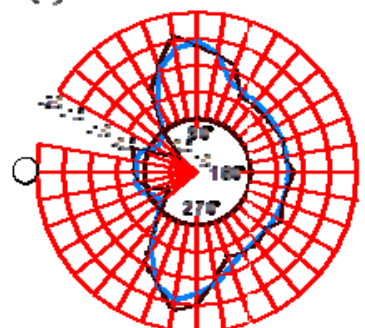

(f) $5 / 8$

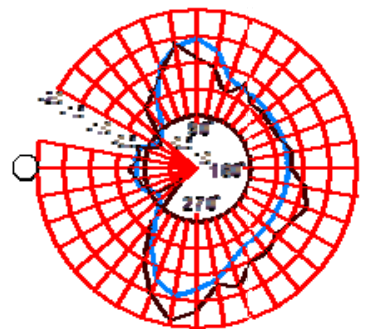

(h) $7 / 8$

Fig. 15. Instantaneous pressure distribution of the controlled circular cylinder at different stages of a vortex shedding cycle 


\subsection{Oscillating fluid force of the controlled cylinders}

The effectiveness of applying this technique to a circular cylinder was then evaluated. Figure 16 shows the Lissajous figures of the oscillating drag and lift coefficients $\mathrm{CD}_{\mathrm{D}}$ and $\mathrm{CL}$ of the cylinders with their flows controlled by the two different methods and that for the single cylinder.

For the forced reattachment method, the center point of the figure has coordinates of $\mathrm{CD}_{\mathrm{D}}=1.1$ and $\mathrm{CL}_{\mathrm{L}}=0.27$, and the amplitude is 0.39 . In this case, a lift force is generated, and the vibration is less than that when using the front rod method.

For the front rod method, the center point of the figure has coordinates of $\mathrm{CD}_{\mathrm{D}}=0.58$ and $\mathrm{CL}_{\mathrm{L}}=0.0$, and the amplitude is 0.50 . In comparison with the single cylinder and the forced reattachment method, the drag force in this case is reduced by over $50 \%$.

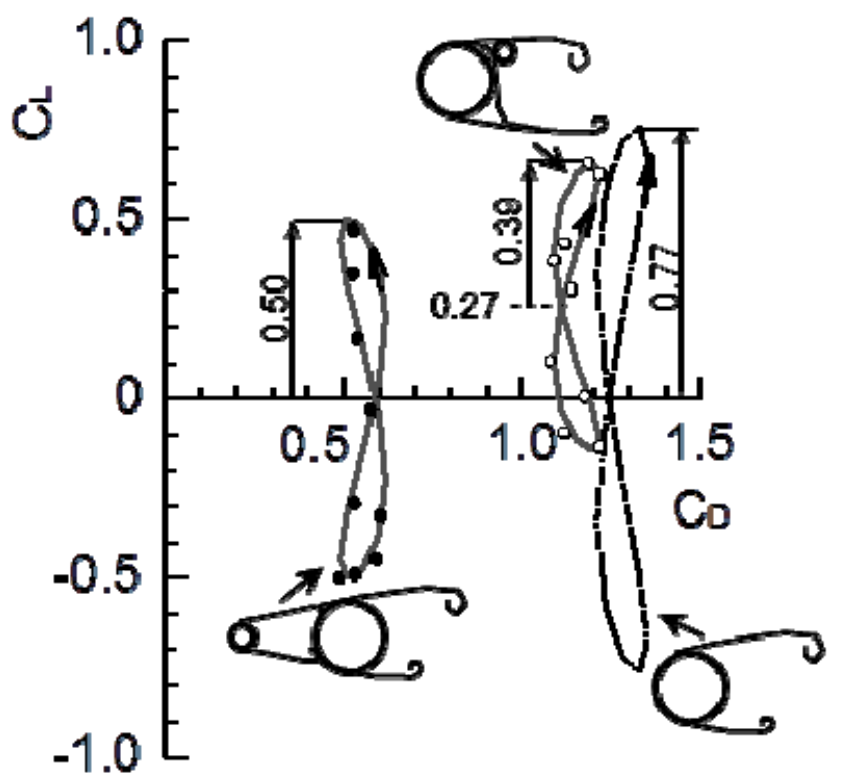

Fig. 16. Lissajous figure

\section{Conclusions}

Two flow control methods, the forced reattachment and front rod methods, were considered in this study. The characteristics of the flow and the oscillating fluid force of a cylinder with the flow controlled by a small rod were investigated experimentally.

When the forced reattachment method was applied, a lift force was generated, and the vibration was less than that in case of the front rod method. In comparison with the single cylinder and the forced reattachment method, the drag force was reduced by over $50 \%$ when the front rod method was used.

\section{References}

1. T. Tsutsui, T. Igarashi, Flow Control around a Circular cylinder by a Small Cylinder, Proc. of the 11th Australasian Fluid Mechanics Conference. Hobart, Australia. pp. 14-18, (1992)

2. T. Igarashi ,T. Tsutsui, Flow Force Acting on a Circular cylinder Controlled by a Small Rod. Proc. of the 3rd JSME-KSME Fluids Engineering Conference. Sendai, Japan. pp. 571-576, (1994)

3. T. Tsutsui, T. Igarashi and K. Kamemoto, Interactive Flow around Two Circular cylinders of Different Diameters at Close Proximity Experiment and Numerical Analysis by Vortex Method, J. Wind Eng. Ind. Aerodyn., 69-71, pp. 279-291,

(1997)

4. T. Tsutsui, T. Igarashi, Flow Control around a Circular cylinder by a Small Cylinder (Properties of Reattachment Jet). Proc. of the 3rd ASME-JSME Joint Fluids Eng. Conf., San Francisco, California, FEDSM99-6943, (1999)

5. T. Tsutsui, T. Igarashi, Drag Reduction of a Circular cylinder in an Air-stream, J. Wind Eng. Ind. Aerodyn., 90, pp. 527-541, (2002)

6. T. Tsutsui, T. Igarashi, Heat Transfer Enhancement of a Circular cylinder, Trans. ASME J. Heat Transfer. 128, pp. 226-233 (2006)

7. T. Igarashi, N. Terachi, Heat transfer enhancement and drag reduction of a flat plate normal to the airstream (flow control using a rod). Heat transfer Asian Res. 27 (2), 99 - 113, (1998)

8. T. Tsutsui, T. Igarashi, H. Nakamura, Drag Reduction and Heat Transfer Enhancement of a Square Prism, JSME Int. Ind. J. B, 44, No. 4, pp. 575 - 583, (2001)

9. T. Igarashi, T. Tsutsui, Enhancement of heat transfer and reduction of drag of a square prism arranged diamond - shape in an air stream, Proc. of the 11th IHTC, Kyongiu, Korea, Vol. 5, pp261 - 266, (1998)

10. H. Nishimura and Y. Taniike, Aerodynamic characteristics of fluctuating forces on a circular cylinder, J. Wind Eng. Ind. Aerodyn, 89, pp. 713723, (2001) 